\title{
Home mechanical ventilation in Hong Kong
}

\author{
C.M. Chu*, W.C. Yü, C.M. Tam ${ }^{\Uparrow}$, C.W. Lam ${ }^{+}$, D.S.C. Hui ${ }^{\S}$, C.K.W. Lai ${ }^{\S}$, \\ on behalf of the Hong Kong Home Ventilation Registry, Hong Kong Thoracic Society
}

Home mechanical ventilation in Hong Kong. C.M. Chu, W.C. Yu, C.M. Tam, C.W. Lam, D.S.C. Hui, C.K.W. Lai, on behalf of the Hong Kong Home Ventilation Registry, Hong Kong Thoracic Society. C ERS Journals Ltd 2004.

ABSTRACT: Home mechanical ventilation (HMV) is increasingly used to treat chronic respiratory failure. This present study was aimed to examine the trend, the disease categories treated and the outcomes of HMV use in Hong Kong.

In the year 2002, all adult respiratory units in Hong Kong were invited to report to a multicentre retrospective survey of HMV use. A total of 249 patients (156 males, mean age $62.7 \pm 13.8 \mathrm{yrs})$ were treated since 1980 , with $197(79 \%)$ continuing with HMV at the time of the survey.

Cumulative number of HMV grew as a cubic function of time since 1980. Currently, there are 2.9 users per 100,000 population. The predominant mode of HMV was noninvasive ventilation by bilevel pressure support ventilators $(n=236)$. Chronic obstructive pulmonary disease accounted for $48.6 \%$ of all cases. The overall 3-yr HMV continuation rate was $66.2 \%$. Death was the main reason for discontinuation.

A rapidly rising trend of home mechanical ventilation use is observed in Hong Kong. In contrast to other series, chronic obstructive pulmonary disease was the major group treated and bilevel pressure support ventilation was the predominant mode chosen. Most patients tolerate home mechanical ventilation reasonably well, with approximately twothirds continuing with its use at $\mathbf{3 6}$ months.

Eur Respir J 2004; 23: 136-141.
*Division of Respiratory Medicine, Dept of Medicine, United Christian Hospital, " Dept of Medicine, Princess Margaret Hospital, "Tuberculosis and Chest Service, Dept of Health, ${ }^{+}$Dept of Medicine, Ruttonjee Hospital, and ${ }^{\S}$ Dept of Medicine and Therapeutics, The Chinese University of Hong Kong, Hong Kong SAR, China.

Correspondence: C.M. Chu, Division of Respiratory Medicine, Dept of Medicine, United Christian Hospital, Hong Kong SAR, China. Fax: 85223472325

E-mail:nncmchu@netvigator.com

Keywords: Asia-Pacific chronic obstructive pulmonary disease

chronic respiratory failure

home mechanical ventilation

Hong Kong

registry

Received: February 172003

Accepted after revision: July 232003
Throughout the world, home mechanical ventilation (HMV) is increasingly employed to treat patients suffering from chronic respiratory failure. Diseases that have been treated by HMV include restrictive thoracic disorders (RTD), neuromuscular disorders, chronic obstructive pulmonary disease (COPD) and various other causes of nocturnal hypoventilation syndrome. The cumulative number of patients being put on HMV has increased tremendously in Europe and the USA [1, 2]. The rapid growth of HMV has been attributed to: 1) increased awareness and experience with the indications and technologies; 2) improved support by machine vendors; 3) pressure to reduce hospital stay; and 4) improved life expectancy in treated patient [2].

Little is known about the prevalence and the growth of HMV use in the Asia-Pacific region. This present study aimed to examine the trend, the disease categories treated and the outcomes of HMV use in Hong Kong, and to explore the differences in the pattern of use with other parts of the world. These data will be useful not only to healthcare providers but also to clinicians in assessing the feasibility and acceptability of long-term HMV in various disease conditions.

\section{Materials and methods}

\section{The survey}

Hong Kong has a population of 6.7 million. More than $95 \%$ of all secondary and tertiary medical services are provided by the publicly funded Hong Kong Hospital
Authority (HKHA). In the year 2002, consultants of respiratory medicine in all adult medical departments of HKHA hospitals were invited to report to a multicentre retrospective survey of their adult patients ( $>18$ yrs of age) who had ever been managed by HMV. Cases that had been managed by more than one institution were counted only once. Only longterm mechanical ventilation conducted at home for $>1$ month was surveyed [3]. Institutionalised chronic ventilator cases were excluded. The series included one patient with Duchenne muscular dystrophy transferred to adult care from paediatric service after the patient had reached $18 \mathrm{yrs}$. The survey captured the following information: demographic data, mode of ventilation (noninvasive or tracheostomy ventilation), underlying disease, indications for HMV, time of starting ventilation, time and reason of stopping ventilation, if any, in the follow-up period. Data was censored at 36 months, as there were not many patients who were followed up beyond this duration.

Most centres had systematically collected data regarding their patients prior to this survey. For the few centres where data was incomplete, a hand search of case records was made. To ensure maximal retrieval of data, especially of previous cases of HMV who may have already died or stopped using HMV at the time of the survey, assistance from the HMV machine vendors were sought to cross-check with their databases.

\section{Statistical analysis}

Data are expressed in mean \pm SD. The cumulative number of patients using HMV (cumulative new cases minus withdrawn 
cases) was plotted against time. Growth in the cumulative number of HMV was modelled by fitting various curves mathematically. Comparisons of characteristics between subgroups were made with the Chi-squared test or one-way analysis of variance where appropriate. The actuarial probability of continuing with HMV [4] was analysed with life-table analysis and Kaplan-Meier plot. Univariate comparisons of the actuarial probability of continuation of $\mathrm{HMV}$ with respect to diagnostic subgroups, indications for HMV, sex and types of HMV were made with a log-rank test. Cox's regression was used to assess the effect of age on the probability of continuation of HMV during follow-up. A multivariate Cox's regression model with stepwise selection was used to extract independent factors affecting the probability of continuation of HMV. A p-value of $<0.05$ was taken as statistically significant.

\section{Results}

There were 249 cases reported to the survey, from 14 centres of adult respiratory medicine. One centre replied it did not have HMV, two other centres did not meet the deadline of the survey, but they had $<10$ cases altogether. Eighty per cent of HMV cases were under the care of six major centres.

The first patient on HMV was started in 1980, and the number of patients receiving HMV remained small until 1997 when this number started to grow tremendously (fig. 1). It was found that the trend of the cumulative number of HMV use was best modelled by a cubic function of time:

Cumulative $\mathrm{n}$ of $\mathrm{HMV}=15.174 t-2.089 t^{2}+0.079 t^{3}-24.056$ (1)

where $t$ is the time (in years) since 1980 (adjusted $\mathrm{R}^{2}=0.971$, $\mathrm{p}<0.0001$ ).

If the growth of HMV numbers continues at the same rate, a cumulative number of $286(95 \%$ confidence interval (CI) 255-317) could be projected by the end of 2004 (fig. 1). There was a doubling in number every $2-3$ yrs recently. At the time of the survey, 197 cases were continuing with HMV. This corresponded to $2.9 \mathrm{HMV}$ users per 100,000 population.

Of the 249 cases notified, there were 156 males $(62.7 \%)$ and 93 females $(37.3 \%)$ with a mean age of $62.7 \pm 13.8$ yrs. The majority $(n=236,94.8 \%)$ were treated by noninvasive ventilation (NIV), with the remaining 13 patients $(5.2 \%)$ receiving tracheostomy ventilation. No particular time trend was noted

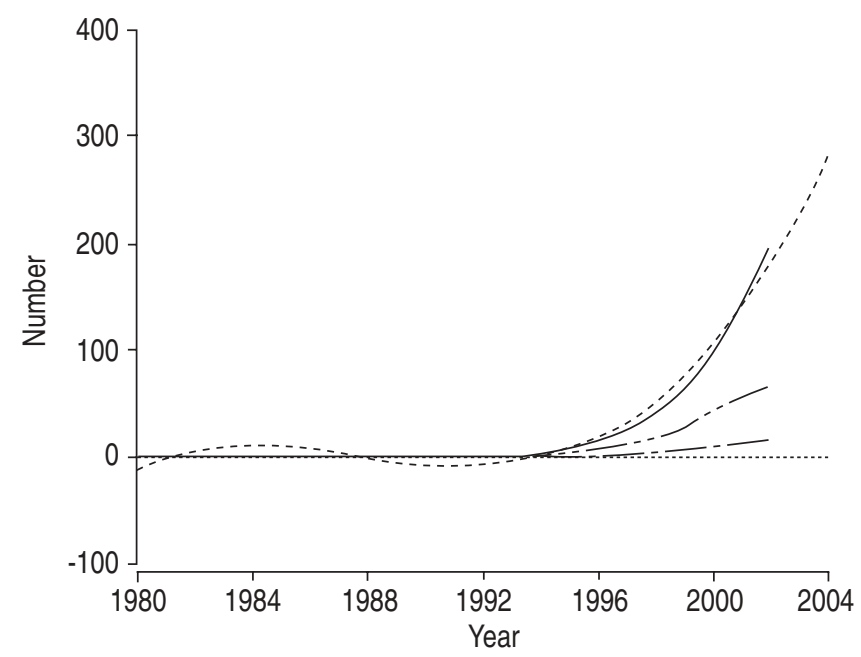

Fig. 1.-New cases (- - - -), withdrawn cases (- - - ) and cumulative number of home ventilation (observed $(-)$ and predicted $(--))$. for tracheostomy ventilation. All NIVs were provided by bilevel pressure-support ventilators. All tracheostomised cases were put on HMV after repeated failures to wean. The disease conditions for which HMV was prescribed are shown in table 1 and the indications for HMV are shown in table 2. Individual diagnoses are merged into the following three subgroups for further analyses: 1) RTD; 2) complicated obstructive sleep apnoea (OSA); and 3) COPD with chronic hypercapnic respiratory failure.

Patients in the RTD $(n=85,34.1 \%)$ were significantly younger and more likely to be tracheostomised than patients in the other subgroups (table 3); 13\% of cases were tracheostomised in this group. The subgroup of complicated OSA had the smallest number of patients $(n=43,17.3 \%)$. It had the highest continuation rate at 36 months, although not statistically significant.

COPD ( $n=121,48.6 \%)$ with chronic hypercapnic respiratory failure was the largest subgroup. The majority were maintained on NIV ( $n=119,98.3 \%)$; only two patients $(1.7 \%)$ were maintained on invasive ventilation. Most of these patients were elected to be put on $\mathrm{HMV}$ as a sequel to acute hypercapnic respiratory failure (AHRF), after suffering from repeated episodes of life-threatening respiratory failure $(65.3 \%)$, or repeated failures to be weaned off ventilation after an acute episode ( $24 \%$; table 2$)$.

An overall actuarial continuation rate of $66.2 \%$ was recorded at 36 months of follow-up. The major cause of discontinuation was death (table 4). The 36-month actuarial continuation rates of the three subgroups are shown in table 3 and the Kaplan-Meier plots are shown in figure 2a. No statistical difference of continuation rate was noted among the three diagnostic subgroups, although there was a trend for a lower continuation rate for COPD and a higher continuation rate for the complicated OSA subgroup. There was no significant difference in the reason for discontinuation in the three subgroups. No difference in the probability of continuation of HMV was noted for sex or the type of HMV.

Indications for commencing HMV were grouped into two subgroups for analyses: sequel to AHRF (failure to be completely weaned or repeated episodes of AHRF, n=188) and other indications (symptomatic hypercapnia, sleep disturbance, failure to respond to continuous positive airway pressure and mixed indications, $\mathrm{n}=61$ ). Patients who were commenced on HMV because of the sequel to AHRF had a significantly lower continuation rate at 36 months $(59 \%)$ versus those started for other reasons $(84 \%$, log-rank test, $\mathrm{p}=0.016$; fig. 2b). Age had a small but statistically significant effect on the probability of continuation; an increase in hazard ratio of $1.25(95 \%$ CI $1.01-1.56, \mathrm{p}=0.043)$ was associated with an increase in each decade of age. On

Table 1.-Diseases treated for home ventilation

\begin{tabular}{lc}
\hline Diagnosis & \\
\hline Restrictive thoracic disorders & \\
Thoracic cage disorders & $38(15.3)$ \\
Post-tuberculous fibrothorax & $9(3.6)$ \\
Neuromuscular disorder & $30(12.0)$ \\
Mixed pathologies and miscellaneous & $8(3.2)$ \\
Complicated OSA/OHS & $11(4.4)$ \\
OHS & $22(8.8)$ \\
COPD: OSA overlap syndrome & $10(4.0)$ \\
Severe OSA, intolerant to CPAP & $121(48.6)$ \\
COPD &
\end{tabular}

Data are presented as n (\%). OSA: obstructive sleep apnoea; OHS: obesity hypoventilation syndrome; COPD: chronic obstructive pulmonary disease; CPAP: continuous positive airway pressure. Percentages add up to $99.9 \%$ because of rounding. 
Table 2. - Primary indications for home ventilation

\begin{tabular}{lccr}
\hline Primary indications & RTD & Complicated OSA/OHS & COPD \\
\hline $\begin{array}{l}\text { Subjects n } \\
\text { Sequel to AHRF }\end{array}$ & 85 & 43 & 121 \\
$\quad$ Failure to be completely weaned & $37(43.5)$ & $20(46.5)$ & $249(24.0)$ \\
$\quad$ Repeated respiratory failure & $19(22.3)$ & $4(9.3)$ & $79(65.3)$ \\
Other indications & $16(18.8)$ & $4(9.3)$ & $102(41.0)$ \\
$\quad$ Symptomatic hypercapnia & $9(10.6)$ & $5(11.6)$ & $11(9.1)$ \\
Sleep disturbance & $0(0)$ & $8(18.6)$ & $1(0.8)$ \\
$\quad$ Failure to respond to CPAP & $4(4.7)$ & $2(4.7)$ & $0(0)$ \\
$\quad$ Mixed indications & & $1(0.8)$ & $8(3.2)$ \\
\hline
\end{tabular}

Data are presented as $\mathrm{n}(\%)$ unless otherwise stated. RTD: restrictive thoracic disorders; OSA: obstructive sleep apnoea; OHS: obesity hypoventilation syndrome; COPD: chronic obstructive pulmonary disease; AHRF: acute hypercapnic respiratory failure; CPAP: continuous positive airway pressure.

Table 3.-Demographics and continuation rates at 36 months (overall and diagnostic subgroups)

\begin{tabular}{|c|c|c|c|c|c|}
\hline Characteristics & All cases & RTD & Complicated OSA/OHS & COPD & p-value $\#$ \\
\hline Subjects $\mathrm{n}$ & 249 & 85 & 43 & 121 & \\
\hline Age yrs & $62.7 \pm 13.8$ & $54.8 \pm 15.1^{\S}$ & $62.8 \pm 14.9$ & $68.1 \pm 9.1$ & $<0.0001$ \\
\hline $\mathrm{M}: \mathrm{F} \mathrm{n}$ & $156: 93$ & $40: 45$ & $33: 10$ & $83: 38$ & 0.001 \\
\hline NIV:tracheostomy n & $236: 13$ & $74: 11^{\S}$ & 43:0 & $119: 2$ & $<0.0001$ \\
\hline Continuation rates at 36 months current study \% & 66.2 & 69.9 & 77.9 & 57.5 & NS \\
\hline Continuation rates at 36 months in Switzerland \% & & $57-78$ & 76 & 49 & \\
\hline Continuation rates at 36 months in France ${ }^{+} \%$ & 73 & $56-80$ & & 56 & \\
\hline
\end{tabular}

Data are presented as mean \pm SD unless otherwise stated. RTD: restrictive thoracic disorders; OSA: obstructive sleep apnoea; OHS: obesity hypoventilation syndrome; COPD: chronic obstructive pulmonary disease; M: male; F: female; NIV: noninvasive ventilation; NS: nonsignificant. \#: p-value refers to comparisons between three subgroups; ": reference [24], $\mathrm{n}=211$; ${ }^{+}$: reference [25], $\mathrm{n}=276 .{ }^{\S}$ : significantly different from other subgroups in post hoc analysis.

multivariate Cox's regression, commencing HMV for the sequel to AHRF was the only independent risk factor for discontinuation during follow-up (hazard ratio $2.57,95 \% \mathrm{CI}$ 1.16-5.74; $\mathrm{p}=0.021$ ). The major cause of discontinuation in this subgroup was death (35 of $44(80 \%))$.

\section{Discussion}

A tremendous increase in the number of HMV in Hong Kong has been noted in recent years. The predominant mode of HMV was NIV, with only a few tracheostomised patients. Bilevel pressure-support ventilators were used in all NIV cases. COPD accounted for $48.6 \%$ of all cases; most patients in this group were started on HMV after repeated weaning failures or repeated episodes of AHRF. The 3-yr HMV continuation rates were $77.9 \%$ for the complicated OSA group, $69.9 \%$ for the restrictive thoracic group and $57.5 \%$ in the COPD group. Patients who were started on HMV because of the sequel to AHRF had a higher discontinuation rate on follow-up, mostly due to death.
To the best of the authors' knowledge, this HMV registry is the first of its type in the Asia-Pacific region. HANCOX et al. [5] reported their experience from a single centre in New Zealand. Although some major centres in Australia provide HMV, no national data could be retrieved from available literature [6]. Similarly, no national data are available from countries in the Asia-Pacific region. In Europe, complete information on HMV can be obtained in Denmark, Spain, Belgium, Switzerland, France and Sweden [7, 8].

The rapidly growing number of patients on HMV in Hong Kong mirrors the development in other countries. In Sweden, it has been estimated that HMV doubled its number in $6 \mathrm{yrs}$ [8]. A similar trend has been recorded in Minnesota, USA [9]. The rate was even higher in this study; the cumulative number increased as a cubic function of time, and the doubling of numbers has been seen recently in 2-3 yrs. The current estimate of HMV use is 2.9 per 100,000 population in Hong Kong, which is lower than most European countries with rates of 4.5-20 per 100,000 [2].

Diseases that have been treated by HMV in this survey are very common conditions in Hong Kong. For example,

Table 4. - Status at last follow-up

\begin{tabular}{|c|c|c|c|c|}
\hline Status at last follow-up & RTD & Complicated OSA/OHS & COPD & All cases \\
\hline Subjects n & 85 & 43 & 121 & 249 \\
\hline Continue with original mode of ventilation & $66(77.6)$ & $36(83.7)$ & $92(76)$ & $194(77.9)$ \\
\hline Switched to an alternative mode of ventilation & $2(2.4)$ & $0(0)$ & $0(0)$ & $2(0.8)$ \\
\hline Died & $11(12.9)$ & $4(9.3)$ & $24(19.8)$ & $39(15.7)$ \\
\hline Refusal of home ventilation & $5(5.9)$ & $3(7)$ & $3(2.5)$ & $11(4.4)$ \\
\hline Home ventilation no longer required & $1(1.2)$ & $0(0)$ & $0(0)$ & $1(0.4)$ \\
\hline Lost to follow-up & $0(0)$ & $0(0)$ & $2(1.7)$ & $2(0.8)$ \\
\hline
\end{tabular}

Data are presented as $\mathrm{n}(\%)$ unless otherwise stated; RTD: restrictive thoracic disorders; OSA: obstructive sleep apnoea; OHS: obesity hypoventilation syndrome; COPD: chronic obstructive pulmonary disease. 

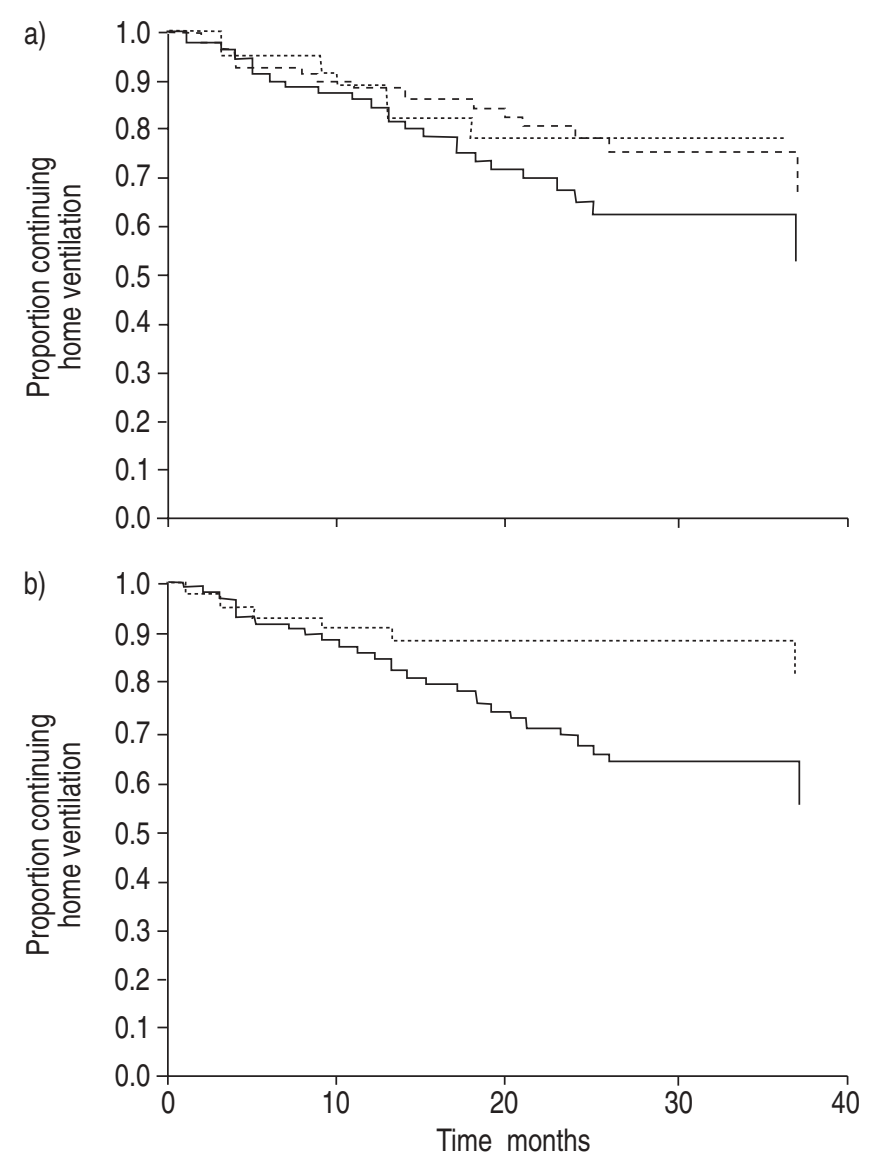

Fig. 2.-Proportion of cases continuing with home ventilation in a) the diagnostic subgroups restrictive thoracic disorders (- - -), obstructive sleep apnoea $(\cdots \cdots \cdots \cdot$, and chronic obstructive pulmonary disease $(-)$, and b) started as a sequel to acute hypercapnic respiratory failure $(-)$ versus other indications $(\cdots \cdots \cdots \cdot)$.

chronic lung conditions developing from late sequelae to tuberculosis (TB) are often seen, i.e. the TB notification rate had reached a high of $\sim 700$ per 100,000 in the 1950 s before declining to $\sim 110$ per 100,000 in the past decade [10]. This compares with 12.7 per 100,000 in England and Wales in 2001 [11]. Similarly, COPD is a common disease in Hong Kong with $10 \%$ of the elderly population having symptoms of chronic bronchitis [12]. A further rise in COPD prevalence would be expected, as an overall smoking rate of $12.4 \%$ is seen in people $>15$ yrs of age [13]. COPD was the fifth leading cause of mortality $(31.4$ per 100,000$)$ in Hong Kong in the year 2001 [14]. In the UK, the mortality rate for bronchitis and allied conditions was 51.3 per 100,000 in males and 38.9 per 100,000 in females in $2001[15,16]$. OSA was also found to be highly prevalent in various sectors of the population [17-20]. The incidence of Duchenne muscular dystrophy is approximately one in 35,000 male births in the USA [21]; exact figures are not available for Hong Kong, but it is estimated to have a prevalence of more than one in 10,000 male births (T.S. Lam, Clinical Genetics Service, Department of Heath, Hong Kong SAR, China; personal communication). Conversely, people in Hong Kong are enjoying longer life expectancy (male 78.0, female 83.9) [22] and the Gross Domestic Product per capita was around US \$24,700 in the year 2001 [23]. Therefore, it would not be surprising if the rapid growth of HMV in Hong Kong continues to be seen.

It has been estimated that home care for tracheostomy ventilation would cost $€ 15$ per patient per day and NIV would cost $€ 9-10$ per patient per day [2]. If the growth of
HMV use continues at the current rate, the cost of home care for HMV would escalate to a minimum of $€ 940,000$ by the end of 2004 (staff cost excluded).

There were important differences between the patterns of HMV use in Hong Kong versus those in other countries, in terms of the mode of ventilation and the proportion of various diagnoses. NIV constitutes the majority of HMV use in Hong Kong $(94.8 \%)$. Bilevel pressure-support ventilation was the only mode chosen for NIV in Hong Kong; no volume-controlled ventilator was used for NIV in Hong Kong. Tracheostomy only accounted for a minority of HMV (5.2\%). In Sweden in 1996, tracheostomy accounted for $25 \%$ of all HMV; volume-controlled ventilators were used in $75 \%$ of patients [8]. Volume-cycled ventilators were the predominant mode of HMV used in Europe in a survey conducted in 1992 [7]. The strikingly low proportion of tracheostomy cases in Hong Kong is probably because of the lack of funding to care for these more complicated and demanding cases at home. The availability and costs of ventilators cannot be excluded as factors influencing the choice of HMV mode. Another possible explanation for the high proportion of pressure-support ventilator use in Hong Kong is that pressure-support ventilation became more readily available and was the preferred mode of ventilation in the majority of patients in the past few years. It was noted that the prescription of pressure-support ventilators also increased considerably in recent years in Sweden [8] and in Switzerland [24].

COPD accounted for $48.6 \%$ of all cases of HMV in Hong Kong. Compared with the $3-29 \%$ of COPD patients in reported surveys of HMV use [4, 5, 8, 24, 25], the current survey has the highest number of COPD. One explanation is that the current study is the most recent; it has been noted that the proportion of COPD cases increases with time in other series [8, 24].

The use of home NIV in stable severe COPD patients is controversial. There have been four randomised studies on the use of NIV in severe stable COPD [26-29]; only one of them has shown favourable results in terms of daytime gaseous exchange, total sleep time, sleep efficiency and quality of life [27]. However, JoNEs et al. [30] have suggested that home NIV may reduce both hospital admissions and clinic visits in severe COPD with hypercapnic respiratory failure. In addition, CLINI et al. [31] have shown that the addition of NIV to long-term oxygen therapy significantly reduces intensive care unit admissions in patients with severe COPD and hypercapnia. Nevertheless, a long-term randomised controlled trial of nocturnal NIV in outpatients with severe COPD has shown that NIV does not affect the natural course of the disease [32]. In the current survey, the majority of the COPD patients were started on HMV after repeated failure to wean or repeated episodes of life-threatening respiratory failure. Although this practice is in keeping with prevailing guidelines [1], more data from randomised controlled trials are clearly needed.

The overall 36-month continuation rate was $66.2 \%$. The $36-$ month continuation rate in COPD was $57.5 \%$, in the RTD group was $69.9 \%$ and the complicated OSA/obesity hypoventilation syndrome (OHS) group was $77.9 \%$. These figures are very similar to those of a Switzerland series, in which the respective 36-month continuation rates for COPD, RTD and OHS subgroups were $49 \%, 57-78 \%$ and $76 \%$, respectively [24]. In a French series, the 36-month continuation rate for COPD was $56 \%$, and rates for RTD and neuromuscular causes were $56-80 \%$ [25]. Direct comparison of outcomes between the current series and the UK series [4] is difficult because of the shorter follow-up period in the current study. However, $50 \%$ of COPD patients discontinued HMV at 19.2 months in the UK series; in the current Hong Kong series, $57.5 \%$ of COPD 
patients were still continuing HMV use at 36 months. In the UK series, $50 \%$ of RTD and neuromuscular patients discontinued HMV at 25.2-51.6 months; in the Hong Kong series, $69.9 \%$ were still continuing at 36 months.

The total number of HMV ever prescribed in Hong Kong is likely to be slightly underestimated in this survey due to the following limitations. Firstly, paediatric patients were not included, and HMV arranged in the private sector was not surveyed. In addition, the possibility of nonrespiratory specialists (e.g. neurologists and rehabilitation physicians) prescribing HMV to their patients cannot be excluded entirely. However, the medico-legal milieu in Hong Kong discourages such practice and such cases have not been identified in the database of the vendors. Lastly, patients who had been on HMV and then withdrawn or died recently may not have been notified. However, the discrepancy between the surveyed number and the true number of HMV use is likely to be small.

Although the first patient started on HMV was recorded in 1980, most hospitals have only started the service in the recent few years. Therefore, the observation was ceased at 36 months, as otherwise the end of the survival curves would only be composed of a small number of patients. Moreover, some parameters of interest were not studied (e.g. quality of life with HMV, lung function data, source of reimbursement, compliance to HMV, type of interface for NIV), since the relevant data was far from complete.

Notwithstanding these limitations, this survey has provided some useful observations. In particular, the observations of the tremendous growth in HMV use and the projected numbers in the next few years are very useful for healthcare planning.

To conclude, a rapidly rising trend of home mechanical ventilation use in chronic respiratory failure has been observed in Hong Kong. It appears that most patients tolerate this form of treatment reasonably well, with approximately twothirds still continuing with home mechanical ventilation use at 36 months. More research is urgently needed in assessing the cost-effectiveness and compliance with this form of treatment in patients with chronic respiratory failure.

\footnotetext{
Acknowledgements. The Hong Kong Thoracic Society would like to dedicate this work to the late Y-M. Tse (1968-2003), who sacrificed herself in the fight against severe acute respiratory syndrome in Hong Kong. The authors would like to thank W.K. Lam and M. Ip for their insightful comments on the manuscript. They also gratefully acknowledge the following physicians for their contribution of patient data: H.S. Chan, J. Chan, K.S. Chan, Y.C. Chan, W. Chen, K.L. Choo, H. Kwok, W.K. Lam, H.M. Ma, T. Mok, C.Y. Tam, K. Tsang, P.Y. Tse, M. Wong, M. Wong, L. Yam and W.W. Yew.
}

\section{References}

1. Goldberg A, Hill N. Clinical indications for non-invasive positive pressure ventilation in chronic respiratory failure due to restrictive lung disease, COPD, and nocturnal hypoventilation: a consensus conference report. Chest 1999; 116: 521-534.

2. Leger P. Organization of long-term mechanical ventilation in Europe. In: Simonds AK, ed. Non-invasive Respiratory Support. A Practical Handbook. 2nd Edn. London, Arnold Publishers, 2001; pp. 271-281.

3. Wicks AR, Menter RR. Long-term outlook in quadriplegic patients with initial ventilator dependency. Chest 1986; 90 : 406-410.
4. Simonds AK, Elliott MW. Outcome of domiciliary nasal intermittent positive pressure ventilation in restrictive and obstructive disorders. Thorax 1995; 50: 604-609.

5. Hancox RJ, Whyte KF, Baxter JM. Home ventilation: the Green Lane Hospital experience. NZ Med J 2000; 113: 500-503.

6. Newton-John HF. Long-term mechanical ventilation of patients in Australia. Med J Aust 1989; 15: 3-6.

7. Fauroux B, Howard P, Muir JF. Home treatment for chronic respiratory insufficiency: the situation in Europe in 1992. Eur Respir J 1994; 7: 1721-1726.

8. Midgren B, Olofson J, Harlid R, Dellborg C, Jacobsen E, Norregaard O. Home mechanical ventilation in Sweden, with reference to Danish experiences. Respir Med 2000; 94: 135-138.

9. Adams AB, Whitman J, Marcy T. Surveys of long-term ventilator support in Minnesota: 1986 and 1992. Chest 1993; 103: $1463-1469$.

10. Tuberculosis and Chest Service. Annual report 2001. Hong Kong Special Administrative Region, Department of Health, 2001.

11. Health promotions agency. Tuberculosis - respiratory and non-respiratory notifications England and Wales: 2001 final and 2002 provisional. www.hpa.org.uk/infections/topics_az/ tb/epidemiology/table2.htm. Accessed May 16, 2003.

12. Lai CKW, Ho SC, Lau J, et al. Respiratory symptoms in elderly Chinese living in Hong Kong. Eur Respir J 1995; 8: 2055-2061.

13. Census and Statistics Department. Thematic Household Survey Report No. 5. Hong Kong Special Administrative Region, Census and Statistics Department, 2001.

14. Department of Health, Hong Kong. Death rates for leading causes, 1981-2001. www.gov.onfo.hk/dh/useful/index.htm. Accessed May 16, 2003.

15. Department of Health, United Kingdom. Indicators of Nations Health: Male death rates by selected causes. www.doh.gov.uk/HPSSS/TBL_A3.htm. Accessed May 16, 2003.

16. Department of Health, United Kingdom. Indicators of Nations Health: Female death rates by selected causes. www.doh.gov.uk/HPSSS/TBL_A4.htm. Accessed May 16, 2003.

17. Hui DS, Chan JK, Ho AS, Choy DK, Lai CK, Leung RC. Prevalence of snoring and sleep-disordered breathing in a student population. Chest 1999; 116: 1530-1536.

18. Ip M, Chung KF, Chan KN, Lam SP, Lee K. Previously unrecognized obstructive sleep apnea in Chinese subjects with essential hypertension. Lung 1999; 177: 391-400.

19. Hui DS, Chan JK, Ko FW, et al. Prevalence of snoring and sleep-disordered breathing in a group of commercial drivers in Hong Kong. Intern Med J 2002; 32: 149-157.

20. Ip MS, Lam B, Lauder IJ, et al. A community study of sleepdisordered breathing in middle-aged Chinese men in Hong Kong. Chest 2001; 119: 62-69.

21. Roland EH. Muscular dystrophy. Pediatr Rev 2000; 21: 233 237.

22. Department of Health, Hong Kong Special Administrative Region. Annual Report 2000/2001. Hong Kong Special Administrative Region, Department of Health, 2000-2001.

23. Census and Statistics Department. Special Report on Gross Domestic Product. Hong Kong Special Administrative Region, Census and Statistics Department, 2002.

24. Janssens JP, Derivaz S, Breitenstein E, et al. Changing patterns in long-term noninvasive ventilation. A 7-year prospective study in the Geneva Lake Area. Chest 2003; 123: 67-79.

25. Leger P, Bedicam JM, Cornette A, et al. Nasal intermittent positive pressure ventilation. Long-term follow-up in patients with severe chronic respiratory insufficiency. Chest 1994; 105: 100-105.

26. Strumpf DA, Millman RP, Carlisle CC, et al. Nocturnal positive-pressure ventilation via nasal mask in patients with 
severe chronic obstructive pulmonary disease. Am Rev Respir Dis 1991; 144: 1234-1239.

27. Meecham Jones DJ, Paul EA, Jones PW, Wedzicha JA. Nasal pressure support ventilation plus oxygen compared with oxygen therapy alone in hypercapnic COPD. $A m$ J Respir Crit Care Med 1995; 152: 538-544.

28. Lin CC. Comparison between nocturnal nasal positive pressure ventilation combined with oxygen therapy and oxygen monotherapy in patients with severe COPD. Am J Respir Crit Care Med 1996; 154: 353-358.

29. Gay PC, Hubmayr RD, Stroetz RW. Efficiency of nocturnal nasal ventilation in stable, severe chronic obstructive pulmonary disease during a 3-month controlled trial. Mayo Clin Proc 1996; 71: 533-542.

30. Jones SE, Packham S, Hebden M, Smith AP. Domiciliary nocturnal intermittent positive pressure ventilation in patients with respiratory failure due to severe COPD: long term follow up and effect on survival. Thorax 1998; 53: 495-498.

31. Clini E, Sturani C, Porta R, et al. Outcome of COPD patients performing nocturnal non-invasive mechanical ventilation. Respir Med 1998; 92: 1215-1222.

32. Casanova C, Celli BR, Tost L, et al. Long-term controlled trial of nocturnal nasal positive pressure ventilation in patients with severe COPD. Chest 2000; 118: 1582-1590. 